\title{
Tight upper bound of the maximum speed of evolution of a quantum state
}

\author{
H. F. Chau* \\ Department of Physics and Center of Computational and Theoretical Physics, University of Hong Kong, Pokfulam Road, Hong Kong
}

(Received 7 March 2010; published 30 June 2010)

\begin{abstract}
I report a tight upper bound of the maximum speed of evolution from one quantum state $\rho$ to another $\rho^{\prime}$ with fidelity $F\left(\rho, \rho^{\prime}\right)$ less than or equal to an arbitrary but fixed value under the action of a time-independent Hamiltonian. Since the bound is directly proportional to the average absolute deviation from the median of the energy of the state $\mathscr{D} E$, one may interpret $\mathscr{D} E$ as a meaningful measure of the maximum information-processing capability of a system.
\end{abstract}

DOI: 10.1103/PhysRevA.81.062133

PACS number(s): 03.65.Xp, 03.67.-a, 89.70.Eg

\section{MOTIVATION AND PRIOR WORK}

It is impossible to build an arbitrarily fast and powerful computer, quantum or classical, because several fundamental physical limits bound the maximum speed of logical operations and the size of memory space [1]. In particular, Bhattacharyya [2], Uhlmann [3], and Pfeifer [4] found that the time $\tau$ needed to evolve a (mixed) state $\rho$ to another state $\rho^{\prime}$ under the action of a time-independent Hamiltonian $H$ is tightly lower-bounded by

$$
\tau \geqslant \tau_{\mathrm{TEUR}} \equiv \frac{\hbar \cos ^{-1}(\sqrt{\epsilon})}{\Delta E} \equiv \frac{g_{\mathrm{TEUR}}(\epsilon) \pi \hbar}{2 \Delta E},
$$

where $\epsilon=F\left(\rho, \rho^{\prime}\right) \equiv\left[\operatorname{Tr}\left(\sqrt{\sqrt{\rho} \rho^{\prime} \sqrt{\rho}}\right)\right]^{2}$ is the fidelity between the two states and

$$
\Delta E=\sqrt{\operatorname{Tr}\left(H^{2} \rho\right)-E^{2}} \equiv \sqrt{\operatorname{Tr}\left(H^{2} \rho\right)-[\operatorname{Tr}(H \rho)]^{2}}
$$

is the standard deviation of the energy of the system. [Actually, the authors of Refs. [3] and [4] considered the more general situation of a time-dependent Hamiltonian whose results can be reduced to Eq. (1) in the time-independent case.] Because of the form of Eq. (1), it is sometimes called the time-energy uncertainty relation (TEUR) bound. Bounds of this type are interesting for they depend only on a modest description of the system. Later on, Margolus and Levitin [5,6] discovered another tight lower bound on the time required to evolve a (pure) state to another state in its orthogonal subspace under the action of a time-independent Hamiltonian $H$. Their bound is inversely proportional to the average energy of the system above the ground state, $E-E_{0}$. Giovannetti et al. [7] extended the Margolus-Levitin (ML) theorem by showing that the time $\tau$ required to evolve between two (mixed) states with fidelity less than or equal to a fixed $\epsilon \in[0,1]$ under the action of a time-independent Hamiltonian is tightly lower-bounded by

$$
\tau \geqslant \tau_{\mathrm{ML}} \equiv \frac{g_{\mathrm{ML}}(\epsilon) \pi \hbar}{2\left(E-E_{0}\right)}
$$

for some smooth function $g_{\mathrm{ML}}$. Although no closed-form expression is known for $g_{\mathrm{ML}}$, it can be approximated to within a few percent of error by [7]

$$
g_{\mathrm{ML}}(\epsilon) \approx\left[g_{\mathrm{TEUR}}(\epsilon)\right]^{2} .
$$

More importantly, Giovannetti et al. found examples in which the ML bound in Eq. (3) is better than the TEUR bound [7]. Note that the smaller the $\tau$, the faster the system can be used for quantum-information processing. In this respect, the tight bounds in Eqs. (1) and (3) show that $\Delta E$ and $E-E_{0}$ are reasonable measures of the maximum possible quantum-information-processing rate of a system [1,5-7].

Here I report another tight lower bound on the time needed to evolve from one (mixed) state to another under the action of a time-independent Hamiltonian such that the fidelity is less than or equal to a fixed value $\epsilon \in[0,1]$. Recall from the discussions of Margolus and Levitin in Refs. [5,6] that the faster the time $\tau$ for a quantum system to evolve between two orthogonal states, the more powerfully the system can process quantum information. In this respect, a lower bound of the time $\tau$ poses a so-called quantum speed limit on the maximum quantuminformation-processing rate of the system. This notion of a quantum speed limit was then generalized by Giovannetti et al. to the study of evolution between two nonorthogonal states [7]. Since the tight evolution-time bound reported here is inversely proportional to the so-called average absolute deviation from the median (AADM) of the energy of the state $\mathscr{D} E$ of the system, I conclude that $\mathscr{D} E$ is also a reasonable measure of the maximum possible quantum-information-processing rate of a system. Finally, I compare this bound with the TEUR bound [2-4] and the ML bound [5-7].

\section{THE EVOLUTION-TIME BOUND}

\section{A. An auxiliary inequality}

I begin by considering an inequality with a simple geometric meaning. The first quadrant of Fig. 1 depicts the (unique) line with the greatest slope that passes through the origin and meets the curve $y=1-\cos x$ at two distinct points (namely, $x=0$ and $x=x_{m}$ ). Clearly, this line is the tangent to the curve at $x=x_{m}$; and its slope $A$ is given by

$$
A=\max \left\{\frac{1-\cos x}{x}: x>0\right\} .
$$

Numerically, I find that

$$
A \approx 0.724611
$$

and

$$
x_{m} \approx 2.33112 \text {. }
$$

\footnotetext{
*hfchau@hkusua.hku.hk
} 


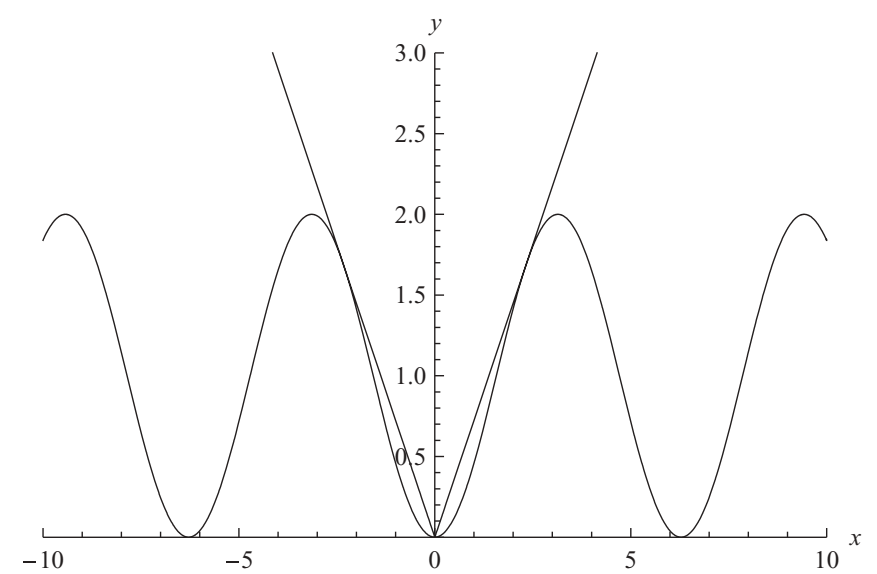

FIG. 1. The curve $y=1-\cos x$ and the broken line $y=A|x|$ defined in the text.

By considering the mirror image of this line with respected to the $y$ axis, it is obvious that

$$
\cos x \geqslant 1-A|x|
$$

for all $x \in \mathbb{R}$. (The geometric meaning of this inequality is apparent from Fig. 1.)

\section{B. The pure-state case}

Now, I may use Margolus and Levitin's argument in Refs. $[5,6]$ to obtain the required bound for the case of pure states. Suppose $|\Phi(0)\rangle=\sum_{j} \alpha_{j}\left|E_{j}\right\rangle$, where the $\left|E_{j}\right\rangle$ 's are the normalized energy eigenvectors of the time-independent Hamiltonian $H$ and $\sum_{j}\left|\alpha_{j}\right|^{2}=1$. Then, under the action of $H$,

$$
\langle\Phi(0) \mid \Phi(t)\rangle \equiv\left\langle\Phi(0)\left|e^{-i H t / \hbar}\right| \Phi(0)\right\rangle=\sum_{j}\left|\alpha_{j}\right|^{2} e^{-i E_{j} t / \hbar} .
$$

In other words, at the time when the system evolves to a state whose fidelity is less than or equal to $\epsilon$ from $|\Phi(0)\rangle$, the real part of Eq. (8) obeys

$$
\left.\left|\sum_{j}\right| \alpha_{j}\right|^{2} \cos \left(\frac{-E_{j} t}{\hbar}\right) \mid \leqslant \sqrt{\epsilon} .
$$

Applying the inequality in Eq. (7) to Eq. (9), I get

$$
1-\frac{A t}{\hbar} \sum_{j}\left|\alpha_{j}\right|^{2}\left|E_{j}\right| \leqslant \sqrt{\epsilon}
$$

Therefore, the earliest time $\tau$ at which $|\Phi(0)\rangle$ evolves to a state whose fidelity is less than or equal to $\epsilon$ from $|\Phi(0)\rangle$ satisfies the inequality

$$
\tau \geqslant \frac{(1-\sqrt{\epsilon}) \hbar}{A \sum_{j}\left|\alpha_{j}\right|^{2}\left|E_{j}\right|} .
$$

By means of the fact that the reference energy level of a system has no physical meaning, I can further strengthen the bound in Eq. (11) as follows: Recall that the function $f(x)=$ $\sum_{j}\left|\alpha_{j}\right|^{2}\left|E_{j}-x\right|$ attains its minimum when $x$ equals $M$, the median of the $E_{j}$ 's with relative frequency of occurrence of
$E_{j}$ equal to $\left|\alpha_{j}\right|^{2}$. More precisely, consider the cumulative distribution function

$$
C(x)=\sum_{j: E_{j} \leqslant x}\left|\alpha_{j}\right|^{2} .
$$

Then

$$
M=\frac{1}{2}\left(\lim _{y \rightarrow 0.5^{-}} C^{-1}(y)+\lim _{y \rightarrow 0.5^{+}} C^{-1}(y)\right) .
$$

(The above assertion can be proven by checking when $d f / d x=0$.) In statistics, the quantity

$$
\begin{aligned}
\mathscr{D} E & \equiv \sum_{j}\left|\alpha_{j}\right|^{2}\left|E_{j}-M\right| \\
& =\operatorname{Tr}\left[\sqrt{(H-M)^{\dagger}(H-M)}|\Phi(0)\rangle\langle\Phi(0)|\right]
\end{aligned}
$$

is known as the AADM of the energy. Thus, I conclude that

$$
\tau \geqslant \tau_{\mathrm{C}} \equiv \frac{(1-\sqrt{\epsilon}) \hbar}{A \sum_{j}\left|\alpha_{j}\right|^{2}\left|E_{j}-M\right|} \equiv \frac{(1-\sqrt{\epsilon}) \hbar}{A \mathscr{D} E} \equiv \frac{g_{\mathrm{C}}(\epsilon) \hbar}{A \mathscr{D} E} .
$$

\section{The mixed-state case}

To extend the above bound to cover the case of mixed states, one simply needs to repeat the argument used by Giovannetti et al. in Ref. [7]: One can always purify the initial and final mixed states. And one may consider a particular choice of the purified states such that the two sets of orthonormal state kets of the ancillary systems used in the purification are identical. Clearly, for other choice of the purified states, the evolution time $\tau$ can never be shorter than the above choice. In addition, the fidelity between this pair of particularly chosen purified states does not exceed the fidelity between the original pair of mixed states. By applying the time bound in Eq. (15) to this particular choice of purified states, one concludes that the bound is also applicable to mixed states [7].

\section{Tightness of the bound}

The time bound in Eq. (15) is certainly tight when $\epsilon=1$. Hence, to show that this bound is tight for all $\epsilon$, I need only to consider the case of $\epsilon<1$. Let me consider the state

$$
|\varphi(0)\rangle=\sqrt{1-\alpha}|0\rangle+\sqrt{\frac{\alpha}{2}}|-\mathcal{E}\rangle+\sqrt{\frac{\alpha}{2}}|\mathcal{E}\rangle,
$$

where

$$
\alpha=\frac{1-\sqrt{\epsilon}}{A x_{m}} \approx 0.592011(1-\sqrt{\epsilon}) \in[0,1] .
$$

Furthermore, $|0\rangle,|\mathcal{E}\rangle$, and $|-\mathcal{E}\rangle$ are normalized energy eigenkets with energies $0, \mathcal{E}$, and $-\mathcal{E}$, respectively. Note that $\langle\varphi(0) \mid \varphi(t)\rangle=1-\alpha+\alpha \cos (\mathcal{E} t / \hbar)$ is a real-valued sinusoidal function of $t$. Besides, it starts to decrease at $t=0$ until $t=\pi \hbar / \mathcal{E}$. Therefore, the earliest time $\tau$ at which $F(|\Phi(0)\rangle,|\Phi(\tau)\rangle)=|\langle\Phi(0) \mid \Phi(\tau)\rangle|^{2} \leqslant \epsilon$ obeys

$$
\sqrt{\epsilon}=1-\alpha+\alpha \cos \left(\frac{\mathcal{E} \tau}{\hbar}\right)=1-\alpha+\alpha \cos \left(\frac{\tau \mathscr{D} E}{\alpha \hbar}\right) .
$$


TABLE I. Comparison between the three lower bounds on $\tau$ for $\epsilon=0$.

\begin{tabular}{|c|c|c|c|c|}
\hline$|\Phi(0)\rangle$ & $\tau$ & $\tau_{\text {TEUR }}$ & $\tau_{\mathrm{ML}}$ & $\tau_{\mathrm{C}}$ \\
\hline$\frac{1}{\sqrt{2}}(|-\mathcal{E}\rangle+|\mathcal{E}\rangle)$ & $\frac{\pi \hbar}{2 \mathcal{E}}$ & $\frac{\pi \hbar}{2 \mathcal{E}}=\tau$ & $\frac{\pi \hbar}{2 \mathcal{E}}=\tau$ & $\frac{\hbar}{A \mathcal{E}} \approx 0.879 \tau$ \\
\hline$|\varphi(0)\rangle$ as defined in Eq. (16) & $\frac{\hbar}{A \alpha \mathcal{E}}$ & $\frac{\pi \hbar}{2 \sqrt{\alpha} \mathcal{E}} \approx 0.876 \tau$ & $\frac{\pi \hbar}{2 \mathcal{E}} \approx 0.674 \tau$ & $\frac{\hbar}{A \alpha \mathcal{E}}=\tau$ \\
\hline$|\varphi(0)\rangle$ as defined in Eq. (16) but & $\frac{7 \pi \hbar}{12 \mathcal{E}}$ & $\frac{\pi \hbar}{\sqrt{4-\sqrt{2}+\sqrt{6}} \mathcal{E}}$ & $\frac{\pi \hbar}{2 \mathcal{E}}$ & $\frac{4 \hbar}{A \mathcal{E}}$ \\
\hline with $\alpha=\frac{4}{4-\sqrt{2}+\sqrt{6}} \approx 0.794$ & & $\approx 0.764 \tau$ & $\approx 0.857 \tau$ & $\approx 0.598 \tau$ \\
\hline$\frac{1}{\sqrt{3}}(|0\rangle+|-\mathcal{E}\rangle+|\mathcal{E}\rangle)$ & $\frac{2 \pi \hbar}{3 \mathcal{E}}$ & $\frac{\pi \hbar}{\mathcal{E}} \sqrt{\frac{3}{8}} \approx 0.919 \tau$ & $\frac{\pi \hbar}{2 \mathcal{E}}=0.75 \tau$ & $\frac{3 \hbar}{2 A \mathcal{E}} \approx 0.988 \tau$ \\
\hline \multirow[t]{2}{*}{$\frac{1}{\sqrt{2 n}} \sum_{k=0}^{n-1}\left[\left|-\left(k+\frac{1}{2}\right) \mathcal{E}\right\rangle+\left|\left(k+\frac{1}{2}\right) \mathcal{E}\right\rangle\right]$} & $\frac{\pi \hbar}{n \mathcal{E}}$ & $\left(n \sqrt{\frac{3}{4 n^{2}-1}}\right) \tau$ & $\left(\frac{n}{2 n-1}\right) \tau$ & $\left(\frac{2}{A \pi}\right) \tau$ \\
\hline & & $\approx 0.866 \tau$ for large $n$ & $\approx 0.5 \tau$ for large $n$ & $\approx 0.879 \tau$ \\
\hline \multirow[t]{2}{*}{$\frac{1}{\sqrt{2 n+1}} \sum_{k=-n}^{n}|k \mathcal{E}\rangle$} & $\frac{2 \pi \hbar}{(2 n+1) \mathcal{E}}$ & $\left(\frac{2 n+1}{4} \sqrt{\frac{3}{n(n+1)}}\right) \tau$ & $\left(\frac{2 n+1}{4 n}\right) \tau$ & $\left(\frac{(2 n+1)^{2}}{2 n(n+1) \pi A}\right) \tau$ \\
\hline & & $\approx 0.866 \tau$ for large $n$ & $\approx 0.5 \tau$ for large $n$ & $\approx 0.879 \tau$ for large $n$ \\
\hline
\end{tabular}

From Eq. (17), I arrive at

$$
\begin{aligned}
(1-\sqrt{\epsilon}) \cos \left(\frac{\tau A x_{m} \mathscr{D} E}{(1-\sqrt{\epsilon}) \hbar}\right) & =(1-\sqrt{\epsilon})\left(1-A x_{m}\right) \\
& =(1-\sqrt{\epsilon}) \cos x_{m} .
\end{aligned}
$$

Note that I have used the fact that the line $y=A x$ intersects with the curve $y=1-\cos x$ at $x=x_{m}$ to arrive at the last line of the above equation. Since $\epsilon<1$, the general solution of Eq. (19) is

$$
\frac{\tau A \mathscr{D} E}{(1-\sqrt{\epsilon}) \hbar}=1+\frac{2 n \pi}{x_{m}}
$$

for all $n \in \mathbb{Z}$. From Eq. (6b), I know that $2 \pi / x_{m}>1$. Therefore, the earliest time $\tau$ at which $|\langle\varphi(0) \mid \varphi(\tau)\rangle|^{2}=\epsilon$ obeys $\tau A \mathscr{D} E /[(1-\sqrt{\epsilon}) \hbar]=1$. Thus, the bound stated in Eq. (15) is tight. After all the discussions above, it is clear that the maximum speed of evolution of a quantum state under the action of a time-independent Hamiltonian $H$ is tightly upper-bounded by $A \mathscr{D} E /[(1-\sqrt{\epsilon}) \hbar]$. And since the reciprocal of the speed of evolution of a quantum system signifies its quantum-information-processing rate [1,5-7], the AADM of the energy $\mathscr{D} E$ is also a reasonable measure of the maximum possible quantum-information-processing rate of a system.

\section{COMPARISON WITH EXISTING MINIMUM-EVOLUTION-TIME BOUNDS}

Now, I start to compare the performance of the three bounds based on $\Delta E, E-E_{0}$, and $\mathscr{D} E$ for fixed values of $\epsilon$. Table I shows the values of these three bounds when $\epsilon=0$ for a few cases in which the $\tau$ 's are known. Clearly, the three bounds complement each other. Moreover, $\tau_{\mathrm{C}}$ is the best whenever $\mathscr{D} E / \Delta E$ and $\mathscr{D} E /\left(E-E_{0}\right)$ are small. This finding is easy to understand. From Eqs. (1), (3), and (15), it is clear that for a fixed value of $\epsilon$, the performances of these three bounds are determined by the ratio $\Delta E: E-E_{0}: \mathscr{D} E$. And the $\tau_{\mathrm{C}}$ bound works best when $\mathscr{D} E \ll \min \left(\Delta E, E-E_{0}\right)$.

Observe that $E-E_{0}$ is the average absolute deviation from the ground-state energy $E_{0}$. (Consequently, the three bounds are in fact based on three different statistical dispersion measures of the eigenvalues of $H$ whose frequencies of occurrence are given by the $\left|\alpha_{j}\right|^{2}$ s.) So, from our earlier discussions on AADM, $\mathscr{D} E \leqslant E-E_{0}$. Furthermore, by a straight-forward application of the Cauchy-Schwarz inequality, one can show that $\mathscr{D} E \leqslant \Delta E$. Note, however, that even though $\mathscr{D} E \leqslant \Delta E$ and $E-E_{0}$, it is still possible for the other two bounds to outperform Eq. (15) because the ratio $g_{\text {TEUR }}(\epsilon): g_{\mathrm{ML}}(\epsilon): 2 g_{\mathrm{C}}(\epsilon) / \pi$ also plays a role in determining which bound is better. But in any case, if the distribution formed by the eigenvalues of $H$ whose frequencies of occurrence are given by the $\left|\alpha_{j}\right|^{2}$,s has a small kurtosis (whose value depends on $\epsilon$, of course), then the time bound due to $\mathscr{D} E$ is better than the other two. As an illustration, I consider the special case in which $\epsilon=0$ and the eigenvalues of $H$ are drawn uniformly from an interval $[a, b]$. The expected values of $\Delta E, E-E_{0}$, and $\mathscr{D} E$ are $(b-a) \sqrt{3} / 6,(b-a) / 2$, and $(b-a) / 4$, respectively. Thus, as the Hilbert space dimension of the state ket increases, the ratio $\tau_{\mathrm{TEUR}}: \tau_{\mathrm{ML}}: \tau_{\mathrm{C}}$ approaches $\sqrt{3}: 1: 4 /(\pi A) \approx 1.732: 1: 1.757$ for a typical state ket $|\Phi(0)\rangle$. So, as a rule of thumb, $\tau_{\mathrm{C}}$ has a good chance of giving a better time bound for $\tau$ when $\epsilon \approx 0$ provided that the kurtosis of the distribution of eigenvalues of $H$ is greater than or equal to the kurtosis of a uniform distribution, namely, $-6 / 5$.

Finally, I study the effect of $\epsilon$ on the performance of the three bounds. Note from Eqs. (1), (4), and (15) that $g_{\text {TEUR }}(0)=g_{\mathrm{ML}}(0)=g_{\mathrm{C}}(0)=1$. Moreover, by differentiating $g_{\mathrm{C}}(\epsilon) / g_{\mathrm{TEUR}}(\epsilon)$ and $g_{\mathrm{C}}(\epsilon) / g_{\mathrm{ML}}(\epsilon)$ with respect to $\epsilon$, I conclude that $g_{\mathrm{C}}(\epsilon) / g_{\mathrm{TEUR}}(\epsilon)$ and $g_{\mathrm{C}}(\epsilon) / g_{\mathrm{ML}}(\epsilon)$ are decreasing and increasing functions of $\epsilon$, respectively. In fact, $\lim _{\epsilon \rightarrow 0^{+}} g_{\mathrm{C}}(\epsilon) / g_{\text {TEUR }}(\epsilon)=0$. In other words, for a sufficiently small value of $\epsilon$, it is likely that $\tau_{\mathrm{TEUR}} \geqslant \tau_{\mathrm{C}} \geqslant \tau_{\mathrm{ML}}$.

\section{CONCLUSIONS}

To summarize, I presented a tight lower bound $\tau_{\mathrm{C}}$ for the time required to evolve between two states with fidelity less than or equal to $\epsilon$ under the action of a time-independent Hamiltonian. This time bound $\tau_{\mathrm{C}}$ works best when the fidelity between the two states, $\epsilon$, is small and the kurtosis of the 
distribution of eigenvalues of $H$ is $\gtrsim-6 / 5$. My result also implies that the AADM of the energy $\mathscr{D} E$ is a reasonable measure of the maximum quantum-information-processing rate of a system.

\section{ACKNOWLEDGMENTS}

I thank C.-H. F. Fung, K. Y. Lee, and H.-K. Lo for their discussions. This work is supported by the RGC Grant No. HKU 700709P of the HKSAR Government.
[1] S. Lloyd, Nature (London) 406, 1047 (2000).

[2] K. Bhattacharyya, J. Phys. A 16, 2993 (1983).

[3] A. Uhlmann, Phys. Lett. A 161, 329 (1992).

[4] P. Pfeifer, Phys. Rev. Lett. 70, 3365 (1993); 71, 306(E) (1993).

[5] N. Margolus and L. B. Levitin, in Proceedings of the Fourth Workshop on Physics and Computation, edited by T. Toffoli,
B. Biafore, and J. Leão (New England Complex Systems Institute, Boston, 1996), pp. 208-211 .

[6] N. Margolus and L. B. Levitin, Physica D 120, 188 (1998).

[7] V. Giovannetti, S. Lloyd, and L. Maccone, Phys. Rev. A 67, 052109 (2003). 